\title{
Le Héros houellebecquien
}

\section{Murielle Lucie Clément}

\section{(2) OpenEdition}

\section{Journals}

\section{Édition électronique}

URL : http://journals.openedition.org/studifrancesi/29868

DOI : $10.4000 /$ studifrancesi.29868

ISSN : 2427-5856

\section{Éditeur}

Rosenberg \& Sellier

\section{Édition imprimée}

Date de publication : 1 avril 2006

Pagination : 91-99

ISSN : 0039-2944

\section{Référence électronique}

Murielle Lucie Clément, «Le Héros houellebecquien », Studi Francesi [En ligne], 148 (XLX | I) | 2006, mis en ligne le 30 novembre 2015, consulté le 19 avril 2021. URL : http://journals.openedition.org/ studifrancesi/29868; DOI : https://doi.org/10.4000/studifrancesi.29868

\section{(c) (i) ()}

Studi Francesi è distribuita con Licenza Creative Commons Attribuzione - Non commerciale - Non opere derivate 4.0 Internazionale. 


\section{Le Héros houellebecquien}

Ces héros que nous côtoyons tout au long des pages, qui sont-ils vraiment? Pouvons-nous en toute conscience et sans restrictions sympathiser avec eux? Quels sont leurs motifs, leurs angoisses, leur vie? Sont-ils aussi éloignés de nous que l'on aurait tendance à le croireau premier abord ou sont-ils au contraire un reflet de nousmêmes?

Dans cette lecture, j'analyserai le caractère des protagonistes houellebecquiens présents dans l'univers diégétique de l'Extension du domaine de la lutte (1994), Les Particules élémentaires (1998), Lanzarote (2000), Plateforme (2001) et l'essai H.P. Lovecraft (1991), par rapport au thème du sang, de l'amour et de l'économie. Je traiterai ensuite du sujet de leur banalité, souvent évoquée par l'auteur lui-même et je me pencherai sur leur filiation en interrogeant leur interchangeabilité.

Je tiens à préciser que ce que je tenterai d'exprimer sur Michel Houellebecq se rapporte uniquement à son œuvre, entièrement aux personnages mis en scène dans les romans. D'autres part, j'éviterai systématiquement des paroles attribuées à l'auteur au cours d'entretiens avec des journalistes ou des confrères. Une seule exception est faite pour les textes d'Interventions qui ont été revus et corrigés par l'auteur en personne.

\section{Le sang}

Dans l'œuvre houellebecquienne, le sang est un leitmotiv récurrent et ambivalent qui influence la vision des héros houellebecquiens. Que ce soit au cours de descriptions oniriques ou littéraires, leur vision, inondée de sang, louvoie aux limites de l'abjection. Abjecte, la fantaisie animalière du narrateur autodiégétique de l'Extension du domaine de la lutte qui présente l'émasculation comme la révélation de la connaissance avec la phrase empruntée aux textes védiques «Tat twam asi». Abjecte par les frissons d'horreur qu'elle engendre chez le lecteur. Frissons d'horreur occasionnés par la succession de miroirs dans laquelle le lecteur se mire sans en être tout à fait conscient. «Tu es ça», lui chuchote chaque ouvrage dans l'habilité de l'auteur à le faire glisser d'un niveau narratif à l'autre. Abjects aussi les rêves qui reflètent, somme toute, la détresse sanglante et rancunière des héros. Mais c'est aussi sanglante et sanguinaire que cette détresse les pousse à la haine de l'Autre et à souhaiter son élimination. Ainsi en est-il de Bruno qui tente d'évincer le Noir de sa classe, mais aussi sa grand-mère. Ainsi en est-il de Michel des Particules élémentaires qui supprimera la race humaine par son équation. C'est aussi le cas du narrateur de l'Extension du domaine de la lutte qui veut faire tuer le Noir par Tisserand. C'est aussi le narrateur de Lanzarote qui annihile l'identité lesbienne de Pam et Barbara et, de même, le cas de Michel de Plateforme qui réduit les habitants du Tiers-monde en esclavage sexuel. Abjecte la misogynie qui se dégage sans ambages du discours aux insultes gratuites. Abjection perverse qui nous fait sourire dans le discours de Bruno, nous ébranle aussi quelquefois lorsqu'il poignarde sa grand-mère en fantasme.

Dans cette optique, les héros, qui appartiennent tous aux cadres, font entendre une tendance raciste, misogyne et xénophobe. A leur décharge, je dois noter qu'ils sont tous très mal dans leur peau et se haïssent tout autant qu'ils détestent leurs semblables. Ils ne peuvent qu'emprunter l'issue sociale offerte par la société diégétique dans laquelle ils vivent. Toutefois, au travers du thème du sang, je peux voir une similitude se déployer au cours des différents romans. Le fil rouge de la solitude ou, pire, 
de l'esseulement des personnages principaux qui les parcourt et dégage une ligne de négativité qui se profile le long de l'œuvre.

Dans l'Extension du domaine de la lutte, c'est le déni de la sexualité avec Tisserand puceau et le narrateur qui tente de s'émasculer. Le déni de la vision est mis en scène dans Les Particules élémentaires avec les yeux crevés, mangés, arrachés, morts. Par exemple, ceux de Di Meloa mais aussi du canari. Lanzarote nous offre le déni de l'homosexualité et de l'amitié avec l'incapacité du narrateur de rester en contact avec Pam et Barbara et la personne de Rudi qui est tout autre que ce qu'il aurait pu croire. Et enfin, dans Plateforme éclate le déni de la pensée par la tête de l'homme au turban écrabouillée et l'attentat qui met fin au plan minutieusement élaboré.

\section{L'amour}

Je pense que l'on peut parler d'absence en ce qui concerne l'amour filial et l'amour paternel. Par ailleurs, ce dernier se colore d'une teinte incestueuse lorsque je considère les sentiments de Jean-Yves pour sa fille Audrey. Les images du père, tout autant que celles de la mère, évoquent des êtres plus en proie à l'égoïsme qu'à l'amour. Une exception forme Marc Clément qui sauve son fils Michel de l'influence néfaste et des mauvais traitements de sa mère. L'amour maternel est absent des romans et les sentiments des mères à l'égard de leurs enfants s'expriment d'une manière égoïste où se décèle l'abject. En cela, Houellebecq contredit son affirmation première sur la douceur des femmes et l'amour maternel. Nous voyons aussi l'occultation de l'amour lesbien au profit du plaisir phallocentrique dans Lanzarote, Plateforme et Les Particules élémentaires.

La relation de Bruno et Michel peut être considérée comme une relation de bonne entente établie d'un commun accord. Les frères se rencontrent sans véritable heurt, mais aussi sans véritable affection l'un pour l'autre. Ils se supportent mutuellement tout au plus en bonne intelligence, étant l'un pour l'autre l'interlocuteur que la nature leur a donné. Comme on dit, ils font avec. Il est toutefois impossible de parler d'amour fraternel.

L'amour, libéré de cette connotation généralement positive qu'on lui accole ordinairement, permet d'entrevoir que les différents liens relationnels, regroupés sous ce substantif, peuvent revêtir une forme tout autant destructrice que constructrice. D'autre part, nous voyons aussi l'incapacité des héros houellebecquiens à aimer, leur impossibilité à trouver le bonheur, l'acharnement de leur destinée à leur refuser la plénitude dans la compagnie de l'Autre. Cet Autre que souvent ils exècrent, qui réside en eux-mêmes et prend racine dans leur haine de soi. Je note une grande similarité dans les représentations des pères et des fils et aussi dans l'image des héros ce qui soulève la question de leur interchangeabilité.

\section{Banalité et interchangeabilité}

Dans Interventions (1998), Houellebecq parle de «l'univers banal rarement décrit (d'autant plus rarement que les écrivains le connaissent mal) qui a surpris dans mes livres - en particulier dans mon roman» Il déclare aussi: «Mes personnages ne sont ni riches ni célèbres; ce ne sont pas non plus des marginaux, des délinquants ni des exclus» ${ }^{1}$. Voici une affirmation qui me paraît injustifiée. Si les personnages 
ne sont pas célèbres, ils ont par contre, de très bons salaires. Le père de Bruno, par exemple, possède plusieurs cliniques spécialisées dans la chirurgie esthétique, ce qui est garant d'un revenu consistant. Raphaël, dans l'Extension du domaine de la lutte est tout de même encore puceau à l'âge de vingt-huit ans, ce qui forme un cas marginal dans une société où la moyenne d'âge du premier rapport sexuel chez les filles comme chez les garçons se situe généralement dans l'adolescence ${ }^{2}$. De plus, il est sur le point de tuer quelqu'un. Par ailleurs, Michel, dans Les Particules élémentaires a une activité sexuelle très limitée: «Sa bite lui servait à pisser. C'est tout»" Christiane et Valérie sont des adeptes du triolisme et de l'échangisme, Pamela et Barbara dans Lanzarote sont lesbiennes non-exclusives, Rudi pédophile, des préférences sexuelles qui peuvent difficilement être qualifiées de majoritaires ${ }^{4}$. Le narrateur de Extension du domaine de la lutte, incite son collègue, Raphaël, à commettre un meurtre ce qui les place dans une position de délinquance indéniable. Le narrateur de Plateforme s'adonne au tourisme sexuel, ce qui n'est pas à la portée de tout le monde, les Thaïes rencontrées sont prostituées, ce qui représente justement un groupe d'exclus et n'est certainement pas représentatif de la population dans son ensemble, les dirigeants d'une agence de voyages font la promotion du tourisme sexuel organisé, et Jean-Yves, un cadre, a des tendances incestueuses et pédophiles.

Ces quelques exemples m'obligent à réfléchir sur la banalité des personnages houellebecquiens et je vois principalement une grande consistance dans la personnalité des héros des différents romans que je résumerai comme suit. Que ce soit Bruno ou les narrateurs ou Michel ou Jean-Yves, ils sont tous en proie à une dépression destructive. D'un autre côté, cette dépression ne les confine nullement dans l'incapacité d'agir. Sans être écrivains confirmés, ils écrivent. Que l'on pense au narrateur de l'Extension du domaine de la lutte et ses fantaisies animalières, à Bruno et ses pamphlets racistes empreints de philosophie, à Michel, son frère, qui confie au papier des équations scientifiques, à Michel de Plateforme qui se met à noircir des rames entières après le drame de l'attentat. Seul le narrateur de Lanzarote n'écrit pas; Rudi, par contre écrit une lettre. Cette aptitude à écrire, est un autre trait familial qui les caractérise et les unifie. Michel et Bruno n’ont de différence que leur quête sexuelle. Crue et directe chez Bruno, elle est sublimée dans la recherche chez Michel. En cela, ils forment un duo parfait. Pile et face d'une même médaille. L'endroit et l'envers du décor cérébral d'un intellectuel névrosé. Car la névrose est un des pivots autour duquel tourne leur personnalité. Le héros anti-héros, immergé dans sa solitude, qui se meut dans la concentration de son égocentrisme aigu, affiné par son égotisme minimaliste.

Un autre point commun est leur intérêt pour l'économie. Ils échafaudent des réflexions et des systèmes sur la question. Jusqu'au narrateur de Lanzarote qui ne peut s'empêcher de faire remarquer à Rudi l'ingéniosité du péage:

$\mathrm{Au}$ sommet, une surprise nous attendait. Une petite guérite semblable à un poste de douane barrait le chemin, surmontée d'un panneau indiquant : «ESPACE NATUREL PROTEGE ». Voilà autre chose, me dis-je. Pour aller plus loin il fallait s'acquitter d'un droit d'entrée de 1000 pesetas, en échange de quoi on vous remettait une petite brochure avertissant de l'entrée dans une réserve mondiale de la biosphère, puis énumérant différentes interdictions. Je lus avec incrédulité que le ramassage d'un caillou pouvait vous valoir une amende de 20.000 pesetas et six mois d'emprisonnement. Quant aux plantes, il ne fallait pas y songer; de toute façon, il n'y avait pas de plantes. Le paysage n'avait pourtant rien de spécialement remarquable ; il était même beaucoup moins beau que ceux que nous avions traversés la veille. Nous nous

(2) Voir à ce sujet J. Mossuz-Lavau, La vie sexuelle en France, Paris, Editions de La Martinière, 2002, pp. 40-47.

(3) M. HouellebecQ, Les Particules élémentaires,
Paris, Flammarion, 1998 p. 28.

(4) Voir à ce sujet J. Mossuz-Lavau, La vie sexuelle en France, Paris, Editions de La Martinière, 2002, pp. 217-221. 
cotisâmes pour payer. «Pas con, leur truc... soufflai-je à Rudi. Tu prends n'importe quel coin un peu paumé, tu laisses se dégrader la route et tu mets un panneau "ESPACE NATUREL PROTĖGÈ ”. Forcément, les gens viennent. Il n'y a plus qu'à installer un péage, et le tour est joué»s.

\section{Filiation diégétique}

Lorsque Houellebecq écrit la préface de la réédition de H.P. Lovecraft, il voit dans ce livre, plutôt qu'un essai, un roman: «Avec le recul, il me semble que j'ai écrit ce livre comme une sorte de premier roman. Un roman à un seul personnage (H.P. Lovecraft lui-même); un roman avec cette contrainte que tous les faits relatés, tous les textes cités devaient être exacts; mais, tout de même une sorte de roman.» ${ }^{6}$

Après cette analyse des héros houellebecquiens, je reconnais en eux le racisme, la xénophobie de H.P. Lovecraftcommentés par Houellebecq: «Paradoxalement, le personnage de Lovecraft fascine en partie parce que son système de valeurs est entièrement opposé au nôtre. Foncièrement raciste, ouvertement réactionnaire, il glorifie les inhibitions puritaines et juge très évidemment repoussantes les «manifestations érotiques directes» ${ }^{7}$. Cependant, comme on peut le lire dans la citation ci-dessus, ils en diffèrent par leur recherche du plaisir sexuel, différentiation à laquelle Michel Djerzinski échappe.

Le narrateur de l'Extension du domaine de la lutte, ce premier «vrai» roman de Houellebecq, nous met en garde: «Mon propos n'est pas de vous enchanter par de subtiles notations psychologiques. Je n'ambitionne pas de vous arracher des applaudissements par ma finesse et mon humour»' N'entend-on pas Houellebecq en écho?

A peu près n'importe quel romancier s'imagine qu'il est de son devoir de donner une image exhaustive de la vie. Sa mission est d'apporter un nouvel « éclairage »; mais sur les faits eux-mêmes il n'a pas absolument le choix. Sexe, argent, religion, technologie, idéologie, répartitions des richesses... un bon romancier ne doit rien ignorer. Et tout cela doit prendre place dans une vision grosso modo cohérente du monde. La tâche, évidemment, est humainement presque impossible, et le résultat presque toujours décevant. Un sale métier?.

Et plus loin: «L'utilisation du vocabulaire scientifique peut constituer un extraordinaire stimulant pour l'imagination poétique» ${ }^{10}$. Une remarque, que non seulement l'Extension du domaine de la lutte mais aussi Les Particules élémentaires illustrent parfaitement.

Michel, le biologiste, démantèle le code génétique ce qui autorise la création d'une nouvelle race humaine et la disparition de la race présente. La phrase suivante se trouve dans H.P. Lovecraft: «L'univers n'est qu'un furtif arrangement de particules élémentaires. Une figure de transition vers le chaos. Qui finira par l'emporter. La race humaine disparaîtra. D'autres races apparaîtront, et disparaîtront à leur tour ${ }^{11}$. Ne dirait-on pas déjà Les Particules élémentaires qui se profilent?

Houellebecq admire H.P. Lovecraft, une admiration qui le conduit à disséquer l'œuvre du «reclus de Providence» et à présenter une analyse de la sociétéque le narrateur de l'Extension du domaine de la lutte nous servira à son tour:

(5) M. HouellebecQ, Lanzarote, Paris, Flammarion, 2000, p. 53.

(6) M. HouellebecQ, Ibid., 1999, p. 6.

(7) M. HouellebecQ, H.P. Lovecraft, Paris, Editions J'ai lu, 1999, p. 28.

(8) M. HouellebecQ, L'Extension du domaine de la lutte, Editions J'ai lu, 1999, p. 16.

(9) ID., H.P. Lovecraft, Paris, Editions J'ai lu, 1999, pp. 61-62, souligné dans le texte.

(10) M. HouellebecQ, H.P. Lovecraft, Paris, Editions J'ai lu, 1999, p. 83.

(11) Ibid., pp. 17-18. 
Le capitalisme libéral a étendu son emprise sur les consciences; marchant de pair avec lui sont advenus le mercantilisme, la publicité, le culte absurde et ricanant de l'efficacité économique, l'appétit exclusif et immodéré pour les richesses matérielles. Pire encore, le libéralisme s'est étendu du domaine économique au domaine sexuel. Toutes les fictions sentimentales ont volé en éclats. La pureté, la chasteté, la fidélité, la décence sont devenues des stigmates ridicules. La valeur d'un être humain se mesure aujourd'hui par son efficacité économique et son potentiel érotique: soit, très exactement, les deux choses que Lovecraft détestait le plus fort ${ }^{12}$.

Sans être aveugle à certains côtés typiques de la personnalité de H.P. Lovecraft, Houellebecq sépare radicalement l'œuvre de l'homme tout en admettant que l'un puisse avoir influencé l'autre: «Paradoxalement, le personnage de Lovecraft fascine [...] Foncièrement raciste, ouvertement réactionnaire» ${ }^{13}$. et plus loin: «Lovecraft a en fait toujours été raciste. Mais dans sa jeunesse ce racisme ne dépasse pas celui qui est de mise dans la classe sociale à laquelle il appartient» ${ }^{14}$ de même que: «Il lui paraît évident que les protestants anglo-saxons sont par nature voués à la première place dans l'ordre social; pour les autres races (que de toute façon il ne connaît que fort peu, et n'a nulle envie de connaître), il n'éprouve qu'un mépris bienveillant et lointain» ${ }^{15}$. Houellebecq déclare que «Le mépris n’est pas un sentiment littéraire très productif; il inciterait plutôt à un silence de bon ton $\gg^{16}$. Il en va autrement lorsque l'on a affaire à des «nègres hideux semblables à des chimpanzés gigantesques ${ }^{17}$ ou des « juifs à face de rat» ${ }^{18}$ car apparemment, la haine décuple les forces et stimule la production littéraire. Houellebecq s'interroge:«Quelle race a bien pu provoquer de tels débordements? $\gg^{19}$. Quelle race et non pas quel individu? C'est moi qui souligne. Il est vrai que «Dans une autre lettre, faisant sinistrement office de précurseur, il [H.P. Lovecraft] préconisera l'utilisation de gaz cyanogène» ${ }^{20}$. De ce passage se dégage le pouvoir de la fascination abjecte comme l'indique Houellebecq: «Utilisant à la fois ses connaissances en génétique et sa familiarité avec les textes sacrés, il [H.P. Lovecraft] construit une synthèse explosive, d'un pouvoir d'abjection inouï» ${ }^{21}$. D'autant plus qu'il précise:«L'évolution du monde moderne a rendu encore plus vivantes les phobies lovecraftiennes» ${ }^{22}$. Houellebecq veut-il parler des «métis monstrueux qui sautillent en se dandinant absurdement $\gg^{23}$. ou, comme pourrait le faire croire le viol de Marylise et les crimes de Di Meola, du monde de la nouvelle Sweet Sixteen: «Dans Sweet Sixteen, une bande de Hell's Angels, simplement décrits au départ comme des voyous ultra violents, finit par se livrer à des rites sacrificiels sur la personne de la fille d'un anthropologue ${ }^{24}$ et de conclure par un hommage: «La source pure de sa poésie: il a réussi à transformer son dégoût de la vie en une hostilité agissante» ${ }^{25}$.

En lisant cet essai, je ne peux m'empêcher de noter la ressemblance entre H.P. Lovecraft et certains des personnages houellebecquiens: «Une haine absolue du monde en général, aggravée d'un dégoût particulier pour le monde moderne. Voilà qui résume bien l'attitude de Lovecraft $»^{26}$. Cette phrase correspond tout à fait à décrire l'attitude du narrateur de l'Extension du domaine de la lutte. Et les «nègres hideux semblables à des chimpanzés gigantesques ${ }^{27}$ me donnent l'impression de lire une phrase de Bruno qui a «commencé à haïr les nègres» ${ }^{28}$ lorsqu'il prit conscience

(12) Ibid., p. 144.

(13) Ibid., p. 28

(14) Ibid., p. 127.

(15) Ibid., p. 128.

(16) Ibid., p. 128.

(17) Ibid., p. 128.

(18) Ibid., p. 131.

(19) Ibid., p. 131.

(20) Ibid., p. 132.
(21) Ibid., p. 140.

(22) Ibid., p. 145, souligné dans le texte.

(23) Ibid, , p. 129.

(24) Ibid., p. 145.

(25) Ibid., p. 150, souligné dans le texte.

(26) Ibid., p. 54.

(27) Ibid., p. 128.

(28) M. HouellebecQ, Les Particules élémentaires, Paris, Flammarion, 1998, p. 238. 
de la petitesse de son sexe. Nous touchons là un des traits de caractère fondamentaux de Bruno: son obsession sexuelle et son racisme. Toutefois, le racisme de Bruno est englobé dans un contexte comique.

Bien que l'auteur ait été surpris de découvrir le racisme de H.P. Lovecraft, son essai H.P. Lovecraft, contre le monde contre la vie, contient en essence l'œuvre entière à naître de la plume de Houellebecq. En effet, dans cet ouvrage, le personnage principal, H.P. Lovecraft, initie la grande saga houellebecquienne de la condition humaine où le narrateur, cette fois-ci incontestablement Houellebecq lui-même, énonce les thèmes qui deviendront familiers dans ses romans ultérieurs et formeront l'idéologie de ses personnages.

Il n'en reste pas moins une distillation presque parfaite des techniques scripturales de H.P. Lovecraft explicitées et regroupées en quelques chapitres dont les titres eux-mêmes forment le plus beau poème que Houellebecq ait probablement composé et qui nous livre l'une des clés pour le lire:

Attaquez le récit comme un radieux suicide

Prononcez sans faiblir le grand Non à la vie

Alors, vous verrez une puissante cathédrale

Et vos sens, vecteurs d'indicibles dérèglements

Traceront le schéma d'un délire intégral

Qui se perdra dans l'innommable architecture des temps

\section{Système économique et politique}

Dans Plateforme, l'implantation des clubs Aphrodite envisagée dans un but thérapeutique relève beaucoup plus de la chasse au trésor, dont le principal bénéficiaire reste l'Occident, que d'un remède à double sens avec profit partagé. Le concept du tourisme sexuel organisé, imaginé par Michel est une instrumentalisation de l'Autre. Un régime totalitaire deviendrait nécessaire pour assurer la survie des «parcs à plaisirs» situés dans les contrées du Tiers-monde où les Occidentaux viendraient exercer leur libido déficiente si le système prôné par Michel était appliqué. En ce qui concerne la mutation génétique élaborée par Michel dans Les Particules élémentaires, son application laisse entrevoir un régime totalitaire similaire à celui qui a sévi dans l'ancienne URSS. D'autre part, le système économique formulé par le narrateur de L'Extension du domaine de la lutte est non seulement erroné, mais sa théorie est pernicieuse puisque grâce à elle il démontre à Raphaël la nécessité de tuer. En outre, il omet de prendre en considération l'éventualité de s'investir avant d'acquérir des profits, ce qui est contraire à la loi du marché. Ces omissions sont, à mon avis, commises pour renforcer l'exposition de la thèse exprimée.

En dernier lieu, j'ai analysé l'essai H.P. Lovecraft, et découvert qu'il contient en essence l'œuvre ultérieure de Houellebecq qui le déclare être son premier roman constitué par un seul personnage. Ce personnage, un écrivain raciste jusqu'à l'obsession, présente dans sa personnalité, des ressemblances évidentes avec les héros houellebecquiens. Pour ma part, je suis tentée de le considéré, dans la saga houellebecquienne, comme le patriarche dont les traits de caractères xénophobes, misogynes et racistes ressurgissent indéniablement dans les héros des romans ultérieurs. C'est en leur sens affiché de l'économie, qu'il se rapporte au sexe ou à l'argent, qu'ils s'éloignent de H.P. Lovecraft. C'est cet élément que Houellebecq a ajouté au personnage de H.P. Lovecraft pris comme modèle pour ses héros. H.P. Lovecraft, l'ancêtre de Bruno, Michel et des narrateurs diégétiques, homo et hétéro. Dans mon analyse des 
personnages, j'ai aussi remarqué leur interchangeabilité dans les romans et d'un roman à l'autre.

Et puis, il est possible de considérer Extension du domaine de la lutte et Les Particules élémentaires comme un roman à thèse, mais Plateforme s'inscrit dans la tradition de l'exotisme comme le conçoit J.-M. Moura. Soulignons l'ambiguïté entre la diégèse et la réalité quotidienne du lecteur et la difficulté pour ce dernier à dissocier les deux. Le lecteur averti, éduqué, au courant des genres littéraires en rit. Il y voit une parodie, des clins d'œil. Il s'amuse: c'est du second degré. Je pense que ce lecteur a tort. Pas de s'amuser. Non, bien au contraire. Mais de ne pas prendre cette littérature au sérieux et de la lire au premier degré, justement.

Peut-être que ma lecture est naïve, mais je vois chaque jour ce qu'il en coûte, à des peuples entiers, de ne pas prendre au sérieux ceux qu'ils considèrent comme les pitres de service. Par exemple, Le Pen en France et récemment, Pim Fortuyn aux Pays-Bas. Ils égaient la scène politique jusqu'au jour où on réalise qu'ils la dominent. Il est alors trop tard. Il en est de même en littérature. Le jour est arrivé de se rendre compte que ce que l'on peut prendre pour des saillies désopilantes n'est autre que les symptômes d'une idéologie profonde axée autour de la xénophobie, du racisme et de la misogynie, exercée à partir d'une tendance autoritaire soutenue par une adhérence au totalitarisme.

\section{Côtéface}

En résumé, le sang est un thème récurrent de l'architecture des romans de Michel Houellebecq où miroitent les grandes questions contemporaines. Le sang dans toute son ambiguïté symbolique, vitale et mortifère, est un des thèmes où l'abject se manifeste le plus. L'abject synonyme de dégoût. Horreur de soi, dégoût de la vie et déni des autres. L'abject qui bien qu'impondérable n'en pèse pas moins, à maintes reprises, sur la pensée des protagonistes jusqu'à les étouffer. Cet abject insaisissable domine le désespoir du narrateur de Extension du domaine de la lutte, envahit la libido de Bruno et annihile celle de Michel dans Les Particules élémentaires et ressurgit avec violence dans Lanzarote et le rêve de Michel de Plateforme. L'abject, charrié par le sang qui devrait oxygéner la vie.

Un autre point que j'ai traité est l'interchangeabilité des héros. Dans chacun d'eux se profilent, à plus ou moins fortes doses, l'égocentrisme, la dépression, la haine de soi et de l'Autre avec une accentuation réelle sur la misogynie, le racisme et la xénophobie. Tous sont de fervents adeptes de la lecture et ils écrivent. A cela, je voudrais ajouter que non seulement ils sont substituables entre eux mais que j'ai décelé des ressemblances indéniables avec la personnalité de H.P. Lovecraft, écrivain pour lequel Michel Houellebecq a la plus grande admiration. Tout ceci, me pousse à voir dans les héros houellebecquiens non pas l'alter ego de l'auteur, mais de H.P. Lovecraft auquel Houellebecq a ajouté l'amour de l'argent et du sexe. Si la mort prend bien des fois le pas sur la vie diégétique, le terme d'amour apparaît mais sans qu'il soit possible d'assurer qu'il ne soit employé pour désigner la sexualité.

De ce qui précède, il ressort que le lien entre l'abject et l'amour ou plus exactement son absence, réside en leur présence dans la psyché des héros. Le fait d'appartenir à une certaine ethnie détermine la place dévolue à l'individu par la société et ce sont les femmes non-occidentales qui occupent dans la diégèse le bas de l'échelle sociale. Il est clair aussi, bien que ce ne soit pas le fond de mon propos, que la loi, la politique et l'économie soient toujours enchevêtrées dans un système qui n'est jamais uniquement social ou économique ou juridique. Tout se tient. Quant à la recherche sur l'amour dans la diégèse, elle était indispensable pour déterminer les caractéristiques des personnages, ce que je pense avoir accompli dans mon optique. 
Dans les romans houellebecquiens, les scènes de sexe récurrentes camouflent une tendance. Elles servent de paravent à l'idéologie des personnages. Elles fascinent par leur crudité et aveuglent tout autant le lecteur qui est de ce fait habilement manipulé vers l'acceptation de l'intolérable. Le sexe chez Michel Houellebecq est une sauce onctueuse, savoureuse, voluptueuse qui enveloppe la pièce de résistance où surnage des particules innommées. Force de l'écrivain que de jeter aux pages l'innommable dans une complexité symphonique. Il est alors compréhensible qu'un lecteur, qui dans sa lecture accorde la prépondérance aux scènes sexuelles, voit dans les romans une littérature pornographique.

\section{Côté pile}

Cette étude pourrait cependant paraître bien partiale si n'était envisagé un autre aspect du problème. En général, lorsque l'on rencontre des individus on ne voit que cinquante pour cent de leur personnalité. Le côté soleil, dirons-nous. Le côté ombre, les trois-quarts du temps, demeure inconnu. Il y a des personnes qui dévoilent un peu plus d'elles-mêmes. Mettons quatre-vingt pour cent dans le meilleur des cas. Le processus peut aussi se produire si l'on fait plus ample connaissance. Mais bien des fois, il restera vingt pour cent cachés; bien souvent parce que la personne ne les connaît pas elle-même. Une sorte de terra icognita de la personnalité. Dans ces vingt pour cent, il y a tous ces penchants, ces tics, ces mesquineries, bref tout ce que personne n'aime voir de soi et souvent des Autres.

Et ces vingt pour cent, c'est justement le pourcentage que Houellebecq a choisi d'éclairer. Alors, bien sûr, les héros ont des tendances xénophobes, racistes, misogynes puisque seuls sont mis en relief ces vingt pour cent d'habitude enfouis au plus profond de l'être. Ces vingt pour cent que nous préférons occulter. Il en résulte que les personnages sont des reflets de personnages; les ombres de la caverne de Platon; ce qui en soi est inquiétant, car ils trahissent l'existence de personnages qui se meuvent dans une réalité étrangère à la diégèse. La nôtre.

Depuis la sortie de Plateforme, il y a eu le onze septembre avec les cris, les pleurs, l'information et la désinformation, mais surtout, la résurgence de la peur atavique de l'Autre et de l'horreur par l'invisible rendu tangible et la haine concrétisée. Le monde entier, chaque individu, a pu apprécier, juger, s'insurger contre ou comprendre les Palestiniens qui allumaient des feux de joie, tiraient hilares des salves en l'air et dansaient pour célébrer les deux tours effondrées. Alors, Michel de Plateforme, un monstre? Je ne le pense pas. Tout au plus humain. Un de ces hommes que le lecteur côtoie journellement dans le bus, dans le métro, au bureau, à l'université, au laboratoire, à l'Elysée. Des hommes normaux, disons à quatre-vingt pour cent, avec ces fameux vingt pour cent que l'on préfère occulter. Ce sont donc ces vingt pour cent que les romans de Michel Houellebecq passent au peigne fin, analysent au microscope et en font ressortir chaque rugosité. Tous ces petits cancers que chacun porte en soi. Qu'il faut traiter! Pour les voir, il faut oser regarder. Ce n'est qu'à ce prix qu'ils ne deviendront pas des gangrènes monstrueuses qui pourriront l'entière société.

Pour ma part, je pense avoir mis en lumière tout au plus vingt pour cent de l'œuvre de Houellebecq. Pour les autres quatre-vingt pour cent, il faudra un autre mémoire, une autre thèse, une biographie ou une autobiographie, voire un roman avec peut-être comme personnage principal Houellebecq. En attendant, je me suis limitée aux personnages.

Je pense, comme je viens de l'énoncer, que les héros houellebecquiens ont des tendances racistes, misogynes et xénophobes. Mais, je pense aussi qu'ils sont un signal d'alarme et nous tendent un miroir où sonder notre image pour déceler si au 
fond de nous ne s'embusque pas une parcelle, aussi infinitésimale soit-elle, de celles de leurs particularités qui nous déplaisent tant. Toutefois, au regard du vingt et un avril deux mille deux en France et du six mai aux Pays-Bas, je m'interroge sur la parution d'ouvrages qui charrient la haine de l'Autre, la misogynie, la xénophobie et le racisme dans une diégèse qui peut facilement être interprétée au premier degré et se révèle alors dangereuse dans son idéologie sous-jacente. 